\title{
Effects of Bifidobacterium animalis subsp. lactis BB-12 on the lipid/lipoprotein profile and short chain fatty acids in healthy young adults: a randomized controlled trial
}

Yujin Lee ${ }^{1}$, Zhaoyong Ba ${ }^{2}$, Robert F. Roberts' ${ }^{2}$ Connie J. Rogers' ${ }^{1}$, Jennifer A. Fleming ${ }^{1}$, Huicui Meng ${ }^{1}$, Emily J. Furumoto ${ }^{2}$ and Penny M. Kris-Etherton ${ }^{1 *}$

\begin{abstract}
Background: Some probiotics have hypocholesterolemic effects in animal studies, which are mediated, in part, by increases in fecal short chain fatty acids (SCFAs). Clinical trials of probiotics on lipids/lipoproteins are inconsistent.

Objective: We examined the effects of Bifidobacterium animalis subsp. lactis BB-12 $2^{\circ}$ (BB-12 $)\left(3.16 \times 10^{9}\right.$ CFUs/day) on lipids and lipoproteins and fecal excretion of SCFAs in healthy adults.

Methods: In a randomized, partially blinded, 4-period, crossover study, 30 adults (11 men, 19 women) aged 18-40 years were randomly assigned to: 1) yogurt smoothie with no BB-12 $(\mathrm{YS}), 2)$ yogurt smoothie with BB-12 $2^{\circ}$ added pre-fermentation (PRE), 3) yogurt smoothie with BB-12 added post-fermentation (POST), 4) BB-12 $2^{\circ}$ containing capsule (CAP). We measured serum lipids/lipoproteins, glucose, insulin, C-reactive protein (CRP), and fecal SCFAs at baseline and after each treatment period.

Results: Total cholesterol (TC), LDL cholesterol (LDL-C), HDL cholesterol (HDL-C), and triglycerides (TGs) did not differ after the PRE, POST, and CAP periods versus the YS or between treatments. Compared to baseline, fecal acetate was significantly increased after the YS $(\Delta=211.89 \pm 75.87 \mu \mathrm{g} / \mathrm{g}, P=0.007)$ and PRE $(\Delta=204.98 \pm 75.70 \mu \mathrm{g} / \mathrm{g}, P=0.009)$ periods. The percent increase in fecal acetate was significantly greater after the YS versus the POST period $(52.2 \pm 13.2 \%$ vs. $24.5 \pm 13.2 \%, P=0.023)$. Fecal total SCFAs, propionate and butyrate did not differ between treatment periods. Fecal total SCFAs were negatively associated with TC $(r=-0.22, P=0.01)$, LDL-C $(r=-0.24, P=0.004)$, age $(r=-0.33, P<0.001)$, and waist circumference $(r=-0.25, P=0.003)$.
\end{abstract}

Conclusions: BB-12 ${ }^{\circ}$ supplementation did not improve lipids, lipoproteins and total and individual fecal SCFAs. Fecal SCFAs were negatively associated with TC, LDL-C, age, and waist circumference.

Trial registration: This trial was registered at clinicaltrials.gov as NCT01399996.

Keywords: Probiotics, BB-12, Lipids, Lipoproteins, SCFAs, Waist circumference

\footnotetext{
*Correspondence: pmk3@psu.edu

'Department of Nutritional Sciences, Pennsylvania State University, 110

Chandlee Laboratory, University Park, PA 16802, USA

Full list of author information is available at the end of the article
} 


\section{Background}

Cardiovascular disease (CVD) is the leading cause of death in the US [1]. Elevated LDL-C causes atherosclerotic CVD and, as such, it is a primary target of lipid lowering therapy [2]. Beyond pharmacotherapy, there is interest in identifying safe, inexpensive and nonpharmacological approaches for LDL-C lowering. Probiotics are defined as 'live microorganisms that when consumed in adequate amounts confer a health benefit on the host' by improving the composition of gut microflora [3]. Previous studies demonstrated that probiotics have beneficial effects on cancer $[4,5]$, diarrhea $[6,7]$, and irritable bowel disease [8,9]. In addition, in our previous paper [10], we showed that probiotics have a potential anti-inflammatory effect by reducing secretion of the pro-inflammatory cytokine, TNF- $\alpha$. In vitro and animal studies demonstrated that specific probiotics have hypocholesterolemic effects through possible mechanisms that include deconjugation of bile acids by bile salt hydrolase [11], production of SCFAs [12], and assimilation of cholesterol and fatty acids into the cell surface of the organism, which makes cholesterol less available for absorption into the circulation [13]. In spite of the consistent results from in vitro and animal studies, the hypocholesterolemic effects of probiotics in human studies are inconsistent. DiRienzo reviewed clinical trials that examined hypocholesterolemic effects of probiotics [14]. Some clinical trials showed that TC and LDL-C decreased after supplementation with specific probiotics [15-17] and in hyperlipidemic children [18], but other studies showed no effects [19-21]. These inconsistent results may be attributed to different strains (Lactobacillus reuteri NCIMB 30242, Enterococcus faecium, Lactobacillus acidophilus La5, Bifidobacterium lactis BB$\left.12^{\circ}\right)$ and doses of probiotics $\left(4.0 \times 10^{6}-3.0 \times 10^{10} \mathrm{CFU} / \mathrm{g}\right.$ daily), delivery matrix (capsule vs. yogurt), study duration (2-60 weeks), and heterogeneous study population (healthy or with hypercholesterolemia or diabetes).

SCFAs are organic fatty acids consisting of 1 to 6 carbon atoms produced through bacterial fermentation of polysaccharide, oligosaccharide, protein, peptide, and glycoprotein precursors in the colon [22, 23]. Acetate, butyrate, and propionate are the predominant SCFAs in humans [24]. Previous studies showed that some probiotics enhance the production of SCFAs, which in turn lowered blood lipid/lipoprotein concentrations [12, 25]. The present study was designed to investigate the effects of Bifidobacterium animalis subsp. lactis BB- $12^{\circ}$ on the lipid profile and fecal SCFA concentrations in healthy young adults. This is the first study to examine the effects of $\mathrm{BB}-12^{\circ}$ on the lipid/lipoprotein profile and fecal SCFA concentrations using duel delivery systems (capsule vs. yogurt) and manufacturing processes (BB$12^{\circ}$ added before vs. after the fermentation process). In addition, the association between metabolic parameters and fecal SCFAs were examined. We hypothesized that BB- $12^{\circ}$ would improve the lipid/lipoprotein profile and increase fecal SCFA concentrations in healthy young adults.

\section{Methods \\ Participants}

Healthy participants ( $n=30 ; 19$ women, 11 men) aged 18 to 40 years were recruited. Details of the inclusion and exclusion criteria were reported previously [10]. In brief, eligibility criteria included: a body mass index (BMI) of $20-35 \mathrm{~kg} / \mathrm{m}^{2}$ and delayed transit time (the amount of time between bowel movements $>24 \mathrm{~h}$ ). Exclusion criteria included smoking, elevated blood pressure $(\geq 140 / 90 \mathrm{~mm} \mathrm{Hg})$, a history of myocardial infarction, stroke, diabetes mellitus, liver disease, kidney disease, and thyroid disease. Participants taking the following supplements/medications were excluded unless they were willing to discontinue for the duration of the study: antibiotics, stool softeners, probiotics, cholesterol-lowering medications and supplements, such as psyllium, fish oil, soy lecithin, niacin, fiber, flax, phytoestrogens, and stanol/sterol supplemented foods, multivitamin, or nonsteroidal anti-inflammatory drugs. Women were excluded if they were lactating, pregnant, or planned to become pregnant during the study. The study protocol was approved by the Institutional Review Board of The Pennsylvania State University. Written informed consent was obtained from all enrolled participants at the screening visit. All the study procedures were conducted at The Pennsylvania State University Clinical Research Center (CRC). The ClinicalTrials.gov identifier is NCT01399996.

\section{Recruitment and screening}

Participants were recruited from March 2012 - October 2013 via flyers posted at university bulletin boards, local newspaper advertisement, and email lists at Penn State University. Potential participants were contacted to conduct the initial telephone screening interview. A trained interviewer asked a list of medical and lifestyle questions. Eligible participants were scheduled for a screening appointment at the CRC. At the screening visit, anthropometric measurements (height, weight, and waist circumference), blood pressure were taken, and a fasting blood sample was collected. Details of the study recruitment and screening were reported previously [10].

\section{Study design and intervention}

This study was a randomized, partially blinded, 4-period, balanced order, crossover design. In the partially blinded design, three treatments with yogurt smoothies were blinded to participants, and the treatment with probiotic capsule was not blinded to participants. However, all 
treatments were blinded to the researchers. Eligible subjects were randomly assigned to one of four treatments: 1 ) yogurt smoothie with no BB-12 (Chr.Hansen, Milwaukee, WI) added (YS); 2) yogurt smoothie with BB-12 added pre-fermentation (PRE); 3) yogurt smoothie with BB-12 added post-fermentation (POST); and 4) one capsule containing BB-12 (CAP). Each treatment period was 4 weeks followed by a 2-week washout period. Participants consumed either one 8-oz serving of a strawberry yogurt smoothie $(220 \mathrm{Kcal})$ or one probiotic-containing capsule daily. Each smoothie/capsule contained $3.16 \times 10^{9}$ CFUs/ day of BB- $12^{\circ}$. The supplemental yogurt smoothies were manufactured at the Department of Food Science at The Pennsylvania State University. Details of the manufacturing process and nutrition information for supplemental drinks were described previously [26]. Daily log sheets and weekly check-ins were completed to verify treatment compliance. In addition, participants were asked to complete 24-h-dietary recalls and physical activity records for 3 days during the last week of each treatment period. Participants agreed to maintain their weight, physical activity level, and refrain from consuming other probiotic-containing products while in the study. Weight, waist circumference, and blood pressure were measured and a fasting blood draw was taken at baseline and at the end of each period at the CRC; frozen fecal samples and completed questionnaires were returned to the CRC at the baseline and endpoint visits.

\section{Blood sample collection}

Whole blood samples were collected by venipuncture on two consecutive days at the end of each treatment period. Participants fasted for $12 \mathrm{~h}$, did not consume alcohol for $48 \mathrm{~h}$, and did not take any vitamins or medication for $24 \mathrm{~h}$ prior to the blood draw. Whole blood was drawn into EDTA-containing tubes, centrifuged at $4{ }^{\circ} \mathrm{C}$ for $15 \mathrm{~min}$. Aliquots of serum and plasma were collected and stored $-80{ }^{\circ} \mathrm{C}$ until the analyses were conducted.

\section{Serum lipid profile}

TC and TGs were measured by enzymatic analysis (Quest Diagnostics, Pittsburgh, PA; coefficient of variation $[\mathrm{CV}]<2 \%$ for both measurements). HDL-C was measured according to the modified heparin-manganese procedure $(\mathrm{CV}<2 \%)$. LDL-C was calculated using the Friedewald equation [LDL-C $=\mathrm{TC}-(\mathrm{HDL}-\mathrm{C}+\mathrm{TG} / 5)]$.

\section{Serum glucose, insulin and CRP}

Glucose was measured by spectrophotometry. Plasma fasting insulin was measured by immunoassay (Quest Diagnostics, Pittsburgh, PA). Serum CRP was measured by latex-enhanced immunonephelometry (Quest Diagnostics; $\mathrm{CV}<8 \%$ ).

\section{Measurement of short chain fatty acids}

Stool samples were collected at baseline and at the end of each treatment period. Participants were provided with a fecal collection kit and asked to immediately store the fecal sample in their home freezer until they visited the CRC. Fecal samples were transported in a cooler with ice packs. The samples were stored at $-80{ }^{\circ} \mathrm{C}$ prior to being aliquoted and extracted. SCFA analysis was performed according to Garcia-Villalba et al [27]. The total SCFAs represents the sum of the fecal excretion of acetate, propionate, isobutyrate, butyrate, isovalerate, valerate, and caproate.

\section{Questionnaires}

Dietary intake was reported using 24-h dietary recalls for 3 days including 2 weekdays and one weekend day. Participants were instructed by trained study staff about how to record all foods and beverages at the baseline visit. Trained staff analyzed the 24-h dietary recalls using Food Processor SQL software (ESHA Research, Salem, OR). Physical activity was recorded using the International Physical Activity questionnaire. In brief, participants were instructed to report their activity by choosing one of nine activity categories for each $15 \mathrm{~min}$ interval (96 interval/day) for 3 days including 2 week days and one weekend day. Total daily physical activity was calculated by averaging the approximate metabolic equivalent of tasks (METs) of performed activities ranging from 3 to 9 over $24 \mathrm{~h}$ [28].

\section{Sample size calculation}

The sample size calculation was based on a previous study [16] in which TC decreased $4.62 \%$ in the probiotic yogurt group. With $\alpha$ set to 0.05 and power set to 0.90 , a sample size of 27 was needed for the present study. After taking into account a $10 \%$ dropout rate, the number of participants required was determined to be 30 .

\section{Statistical analysis}

Statistical analyses were performed using SAS (version 9.3; SAS Institute, Cary, NC). Normality for each variable was assessed using the univariate procedure (PROC UNIVARIATE) to check skewness. Log transformation was used if a variable was not normally distributed. All the values are presented as mean \pm SEM. Baseline characteristics of participants were compared by sex using a twosample $t$-test (PROC TTEST). $P<0.05$ was considered significant. Differences between treatment groups were assessed by analysis of variance using mixed procedure (PROC MIXED). Treatment, visit and sex were considered as fixed effects and baseline values, age, and BMI were adjusted as covariates. Participant ID was considered as a random effect. Interactions between sex and treatment group and between treatment and visit (carryover effect) were included in the model, and there were no significant 
interactions in the model, so they were not included in the final model. Tukey-adjusted $P$ values were used for post hoc comparisons between groups. The adjusted $P<0.05$ was considered significant. Change scores were calculated by subtracting baseline values from values after each treatment. Percentage change scores were calculated by dividing change scores by baseline values, then multiplying the result by 100 . The percentage change in fecal acetate was square root transformed. For glucose, insulin, and CRP, Kruskal-Wallis $\mathrm{H}$ test was used to compare differences among groups (PROC NPAR1WAY). For the correlation analysis, the Pearson correlation coefficient was used for normally distributed data, and the Spearman correlation coefficient was used for propionate, glucose, insulin, insulin resistance index (HOMA-IR), and CRP.

\section{Results}

\section{Baseline characteristics}

The study design and flow of participants were reported previously [10]. Of thirty-six participants, six participants dropped out at baseline. Data for thirty participants were analyzed (Fig. 1). Anthropometric measurements, blood pressure, metabolic parameters, physical activity index (METs) at the baseline visit are shown in Table 1 . The participants (19 women and 11 men) were on average $28.2 \pm 6.4$ y of age with a mean BMI of $24.2 \pm 2.6 \mathrm{~kg} / \mathrm{m}^{2}$. BMI, waist circumference, blood pressure and biochemical markers were within normal ranges for both men and women.

\section{Nutrient composition of treatments}

Nutrient composition of the yogurt smoothie and BB- $12^{\circ}$ containing capsule is presented in Table 2. The yogurt smoothie contained milk fat, milk solids non-fat, strawberry puree, pectin, corn syrup solids, sugar, and water. Participants consumed 8 oz. of yogurt smoothie/day that provided 220 kcals. The yogurt smoothie contained $2.5 \mathrm{~g}$ of fat (1.5 g of saturated fat), $10 \mathrm{mg}$ of cholesterol, $90 \mathrm{mg}$ of sodium, $45 \mathrm{~g}$ of carbohydrate ( $1 \mathrm{~g}$ of fiber and $31 \mathrm{~g}$ of sugar), and $7 \mathrm{~g}$ of protein. The nutrient composition of YS, PRE, and POST was identical except for the amount of $\mathrm{BB}-12^{\circ}$. The YS did not contain $\mathrm{BB}-12^{\circ}$ while the PRE and POST yogurt smoothies contained $3.16 \times 10^{9} \mathrm{CFUs}$ of BB- $12^{\circ}$. The CAP contained $3.16 \times 10^{9} \mathrm{CFUs}$ of BB- $12^{\circ}$ that provided $<2 \mathrm{kcals}$ from the gelatin capsule.

\section{Dietary intake}

Energy intake did not differ by treatment period although $8 \mathrm{oz}$. of yogurt smoothie $(220 \mathrm{kcal} /$ day $)$ was added to their usual diet during YS, PRE and POST periods (Table 3). Consistently, there were no differences between the intervention periods with regard to intake of carbohydrate, protein, fat, fiber, and cholesterol. Compared with baseline, total calories carbohydrate, protein, fat, fiber and cholesterol intakes did not increase during any treatment period.

\section{Anthropometrics and blood pressure}

There were no significant differences in anthropometric variables and blood pressure between intervention

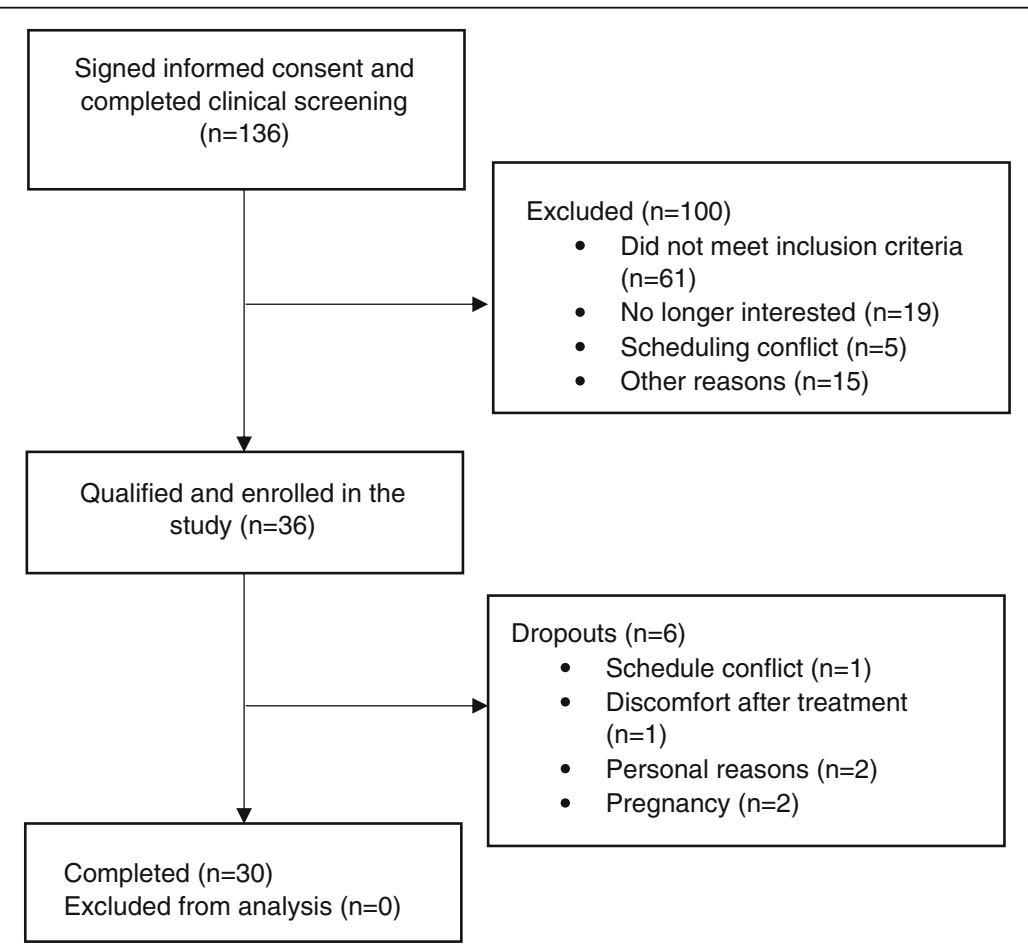

Fig. 1 Participant recruitment flow 
Table 1 Baseline characteristics of participants ${ }^{a}$

\begin{tabular}{|c|c|c|c|}
\hline & Men & Women & Total \\
\hline$n$ & 11 & 19 & 30 \\
\hline Age, y & $29.3 \pm 7.0$ & $27.6 \pm 6.2$ & $28.2 \pm 6.4$ \\
\hline $\mathrm{BMI}, \mathrm{kg} / \mathrm{m}^{2}$ & $23.9 \pm 2.2$ & $24.4 \pm 2.8$ & $24.2 \pm 2.6$ \\
\hline Waist circumference, cm & $85.3 \pm 7.5$ & $85.0 \pm 7.8$ & $85.1 \pm 7.6$ \\
\hline Systolic blood pressure, $\mathrm{mm} \mathrm{Hg}^{*}$ & $114.6 \pm 7.1$ & $102.4 \pm 9.0$ & $107 \pm 10$ \\
\hline Diastolic blood pressure, $\mathrm{mm} \mathrm{Hg}$ & $75.5 \pm 7.2$ & $71.9 \pm 6.3$ & $73 \pm 7$ \\
\hline $\mathrm{TC}, \mathrm{mg} / \mathrm{dL}$ & $160.3 \pm 29.9$ & $168.5 \pm 28.4$ & $166 \pm 29$ \\
\hline $\mathrm{HDL}-\mathrm{C}, \mathrm{mg} / \mathrm{dL}^{*}$ & $47.8 \pm 7.1$ & $60.6 \pm 11.4$ & $56 \pm 12$ \\
\hline $\mathrm{LDL}-\mathrm{C}, \mathrm{mg} / \mathrm{dL}$ & $92.9 \pm 27.1$ & $91.4 \pm 25.2$ & $92 \pm 25$ \\
\hline $\mathrm{TC} / \mathrm{HDL}-\mathrm{C}$ ratio & $3.4 \pm 0.9$ & $2.9 \pm 0.7$ & $3.1 \pm 0.8$ \\
\hline TG/HDL-C ratio ${ }^{c}$ & $2.1 \pm 0.9$ & $1.4 \pm 0.5$ & $1.7 \pm 0.7$ \\
\hline $\mathrm{TGs}, \mathrm{mg} / \mathrm{dL}$ & $97.6 \pm 33.7$ & $82.7 \pm 27.8$ & $88 \pm 30$ \\
\hline Glucose, mg/dL ${ }^{b}$ & $85.5(82.0-90.0)$ & $87.3(84.0-91.0)$ & $87.0 \pm 7.4$ \\
\hline Insulin, $\mathrm{mU} / \mathrm{L}^{\mathrm{b}}$ & $3.5(1.0-6.5)$ & $4.5(2.5-7.0)$ & $4.3(2.0-7.0)$ \\
\hline HOMA-IR & $0.9 \pm 0.7$ & $1.3 \pm 1.1$ & $1.1 \pm 1.0$ \\
\hline$C R P, m g / L^{b}$ & $0.5(0.3-1.4)$ & $0.8(0.3-1.8)$ & $0.7(0.3-2.0)$ \\
\hline Physical activity (METs) b & $1.7(1.6-1.8)$ & $1.8(1.6-1.9)$ & $1.7(1.6-1.9)$ \\
\hline
\end{tabular}

${ }^{a}$ Values are means \pm SDs; TC, total cholesterol; HDL-C, high-density lipoprotein cholesterol; LDL-C, low-density lipoprotein cholesterol; TGs, triglycerides; HOMA-IR, homeostatic model assessment-estimated insulin resistance; hs-CRP, high-sensitivity C-reactive protein; MET, metabolic equivalent of task

${ }^{b}$ Median; interquartile range in parentheses; *Significant difference between men and women $(P<0.05)$

periods. Compared with baseline, there were no significant changes in anthropometric variables and blood pressure after all of the treatment periods (Data not shown).

Associations between SCFAs and metabolic parameters are shown in Table 4. A negative correlation was shown between BMI and butyrate excretion $\left(r_{\mathrm{p}}=-0.18, p=\right.$ $0.04)$. In addition, there was a negative correlation between waist circumference and the total SCFAs $\left(r_{\mathrm{p}}=-0.25\right.$, $p=0.003)$, acetate $\left(r_{\mathrm{p}}=-0.23, p=0.006\right)$, and butyrate $\left(r_{\mathrm{p}}=-0.25, p=0.003\right)$ excretion. Age was negatively correlated with the fecal excretion of total SCFAs $\left(r_{\mathrm{p}}=-0.33, p<0.001\right)$, acetate $\left(r_{\mathrm{p}}=-0.24, p=0.005\right)$

Table 2 Nutrient composition of the yogurt smoothies ${ }^{a}$ (YS, PRE, and POST) and the BB- $12^{\circ}$ containing capsule (CAP)

\begin{tabular}{|c|c|c|c|}
\hline & YS & PRE/POST & CAP \\
\hline Calories, kcals & 220 & 220 & $<2$ \\
\hline Total Fat, g & 2.5 & 2.5 & - \\
\hline Saturated fat, $g$ & 1.5 & 1.5 & - \\
\hline Cholesterol, mg & 10 & 10 & - \\
\hline Sodium, mg & 90 & 90 & - \\
\hline Total Carbohydrate, g & 45 & 45 & - \\
\hline Dietary Fiber, g & 1 & 1 & - \\
\hline Sugar, $g$ & 31 & 31 & - \\
\hline Protein, $g$ & 7 & 7 & - \\
\hline BB-12 (CFUs) & - & $3.16 \times 10^{9}$ & $3.16 \times 10^{9}$ \\
\hline
\end{tabular}

${ }^{\mathrm{a} P e r} 8$ oz. of serving butyrate $\left(r_{\mathrm{p}}=-0.31, p<0.001\right)$, and propionate $\left(r_{\mathrm{s}}=-0.39\right.$, $p<0.001)$

\section{Serum parameters}

Compared with baseline, in response to the treatments, there were no significant changes in TC, HDL-C, nonHDL-C, TGs, TC: HDL-C, insulin, glucose, HOMA-IR, and CRP. In addition, the serum parameters did not differ by the treatment period (Table 5).

Serum TC, LDL-C, and non-HDL-C were negatively correlated with all of the fecal markers $(p<0.05$ for all cases; Table 4).

\section{Fecal short chain fatty acids}

Compared to baseline, fecal excretion of total SCFAs, propionate and butyrate remained unchanged after all treatment periods, whereas acetate excretion was significantly increased after the YS $(\Delta=211.89 \pm$ $75.87 \mu \mathrm{g} / \mathrm{g}, p=0.007$; Table 6) and PRE $(\Delta=204.98 \pm$ $75.70 \mu \mathrm{g} / \mathrm{g}, p=0.009)$ periods. Percentage change was used to measure the magnitude of change between groups; there was a greater increase in acetate excretion after the YS period compared with the POST period. $\left(52.2 \pm 13.2 \%\right.$ vs. $24.5 \pm 13.2 \%, P_{\text {adj }}=0.023$; Fig. 2$)$. However, there was no significant percentage change in the excretion of total SCFAs, propionate and butyrate between the treatment periods (Data not shown). 
Table 3 Dietary intake of participants at baseline and after 4 weeks of the YS, PRE, POST, or CAPSULE treatment period ${ }^{a, b}$

\begin{tabular}{|c|c|c|c|c|c|c|}
\hline & $\begin{array}{l}\mathrm{BL} \\
(n=30)\end{array}$ & $\begin{array}{l}Y S \\
(n=25)\end{array}$ & $\begin{array}{l}\text { PRE } \\
(n=25)\end{array}$ & $\begin{array}{l}\text { POST } \\
(n=26)\end{array}$ & $\begin{array}{l}\text { CAP } \\
(n=26)\end{array}$ & $P$ Value* \\
\hline Total calories, kcal/d & $2472 \pm 152$ & $2224 \pm 164$ & $2338 \pm 161$ & $2092 \pm 165$ & $2321 \pm 162$ & 0.43 \\
\hline Carbohydrate, g/d & $305 \pm 21$ & $287 \pm 23$ & $291 \pm 22$ & $270 \pm 23$ & $305 \pm 21$ & 0.79 \\
\hline Carbohydrate, \% of energy & $49.3 \pm 1.5$ & $50.5 \pm 1.6$ & $50.9 \pm 1.6$ & $51.8 \pm 1.6$ & $50.6 \pm 1.6$ & 0.75 \\
\hline Protein, $\mathrm{g} / \mathrm{d}$ & $97.3 \pm 6.7$ & $94.6 \pm 7.3$ & $95.8 \pm 7.2$ & $84.4 \pm 7.3$ & $93.3 \pm 7.2$ & 0.67 \\
\hline Protein, $\%$ of energy & $15.8 \pm 0.6$ & $17.0 \pm 0.7$ & $16.7 \pm 0.7$ & $16.2 \pm 0.7$ & $16.0 \pm 0.7$ & 0.44 \\
\hline Fat, $\mathrm{g} / \mathrm{d}$ & $96.9 \pm 6.1$ & $79.2 \pm 6.7$ & $87.0 \pm 6.5$ & $75.9 \pm 6.7$ & $90.0 \pm 6.6$ & 0.09 \\
\hline Fat, \% of energy & $35.2 \pm 1.2$ & $32.9 \pm 1.3$ & $33.2 \pm 1.3$ & $32.5 \pm 1.3$ & $34.4 \pm 1.3$ & 0.33 \\
\hline Fiber, g/d & $21.3 \pm 1.7$ & $21.9 \pm 1.8$ & $21.4 \pm 1.8$ & $21.0 \pm 1.8$ & $24.4 \pm 1.8$ & 0.39 \\
\hline Cholesterol, mg/d & $310 \pm 25$ & $288 \pm 27$ & $276 \pm 27$ & $283 \pm 27.4$ & $262 \pm 27$ & 0.71 \\
\hline
\end{tabular}

${ }^{\mathrm{a}}$ Values are means \pm SDs; BL, baseline; YS, yogurt smoothie with no BB-12; PRE, yogurt smoothie with BB-12 ${ }^{\circ}$ added pre-fermentation; POST, yogurt smoothie with BB- $12^{\circ}$ post-fermentation

${ }^{b}$ Statistical analyses were calculated using sex, treatment as between subject factor and randomization ID as the within-subject factor and age and BMI as covariates

${ }^{*} P$ values are for the main effect of treatment

\section{Discussion}

The present study assessed the effect of $\mathrm{BB}-12^{\circ}$ on the lipid/lipoprotein profile and fecal SCFAs excretion. We found that daily BB-12 consumption for 4 weeks did not affect lipid or lipoprotein concentrations (Table 5). The observed a lack of a BB- $12^{\circ}$ effect on the lipid/lipoprotein profile agrees with a prior study in which a mixture of BB-12 and L. acidophilus La5 was tested [19]. In that study, 156 overweight men and women over $55 \mathrm{y}$ randomly received one of the four treatments for 6 weeks: 1) probiotic yogurt plus probiotic capsules; 2) probiotic yogurt plus placebo capsules; 3 ) control milk plus probiotic capsules; and 4) control milk plus placebo capsules. The results showed that neither probiotic yogurt nor probiotic capsule alter the concentrations of TC, LDL-C, HDL-C or TG. In contrast, Ejtahed et al [16] found a $4.54 \%$ decrease in $\mathrm{TC}$ and a $7.45 \%$ decrease in LDL-C in 60 individuals with type 2 diabetes mellitus. These findings differ from what we found, however, the participant characteristics were different between the two studies. In the study by Ejtahed et al., the participants were 51 year of age, had type 2 diabetes and a mean baseline TC and LDL-C of $195 \mathrm{mg} / \mathrm{dL}$ and $117 \mathrm{mg} / \mathrm{dL}$, respectively, whereas our participants did not have type 2 diabetes, were much younger (mean age of 28 years) and baseline TC and LDL-C were $166 \mathrm{mg} /$ $\mathrm{dL}$ and $92 \mathrm{mg} / \mathrm{dL}$, respectively. These findings suggest that the lipid lowering effect of probiotic may be limited to populations with borderline high or high TC and LDL-C. In addition, the lack of a BB- $12^{\circ}$ effect on lipid/ lipoprotein concentrations may be attributable to the probiotic strain we used because studies with different probiotic strains found different results $[15,17,29,30]$. Specifically, studies of Enterococcus faecium demonstrated that TC and LDL-C concentrations decreased after consumption of the probiotic $[15,17,30]$.

In the present study, there were no differences in total fecal SCFAs between the treatment periods. However, fecal acetate excretion significantly increased after the YS and PRE periods compared to baseline (Table 6). It is noteworthy that the fecal acetate excretion did not increase after the POST period compared to baseline. The amounts of BB- $12^{\circ}$ in the PRE and POST were not different and the ingredients in the yogurt smoothie were identical (Table 2). In addition, dietary intake of participants did not differ by the treatment period (Table 3). We surmised further that the manufacturing process (PRE vs. POST) of the yogurt smoothie may

Table 4 Correlations between metabolic parameters and short chain fatty acids at all time points ${ }^{a}$

\begin{tabular}{lllllllllllllll}
\hline & TC & HDL & LDL & N-HDL & TG & TC:HDL & TG:HDL & Glucose & Insulin & HOMA & CRP & Age & BMl & WC \\
\hline Total SCFAs & $-0.22^{*}$ & -0.001 & $-0.24^{*}$ & $-0.24^{*}$ & -0.09 & -0.13 & -0.05 & 0.12 & 0.16 & 0.16 & -0.12 & $-0.33^{* *}$ & -0.16 & $-0.25^{* *}$ \\
Acetate & $-0.18^{*}$ & 0.04 & $-0.24^{* *}$ & $-0.22^{*}$ & -0.03 & -0.12 & -0.004 & 0.13 & $0.18^{*}$ & $0.19^{*}$ & -0.14 & $-0.24^{* *}$ & -0.16 & $-0.23^{* *}$ \\
Butyrate & $-0.27^{* *}$ & 0.01 & $-0.30^{* *}$ & $-0.29^{* *}$ & -0.12 & $-0.17^{*}$ & -0.07 & 0.05 & 0.08 & 0.08 & -0.08 & $-0.31^{* *}$ & $-0.18^{* *}$ & $-0.25^{* *}$ \\
Propionate & $-0.25^{* *}$ & -0.08 & $-0.22^{* *}$ & $-0.22^{*}$ & -0.08 & -0.12 & -0.04 & $0.17^{*}$ & $0.20^{*}$ & $0.21^{*}$ & 0.05 & $-0.39^{* *}$ & 0.07 & -0.10 \\
\hline
\end{tabular}

a Pearson coefficients were used for total SCFAs, acetate, butyrate; spearman coefficients were used for propionate, glucose, insulin, HOMA-IR, and hs-CRP; TC, total cholesterol; HDL-C, high-density lipoprotein cholesterol; LDL-C, low-density lipoprotein cholesterol; N-HDL, non-high-density lipoprotein cholesterol; TGs, triglycerides; HOMA-IR, homeostatic model assessment-estimated insulin resistance; CRP, C-reactive protein; BMI, body mass index; WC, waist circumference. ${ }^{*} P<0.05,{ }^{* *} P<0.01$ 
Table 5 Serum parameters of participants at baseline and after 4 weeks of the YS, PRE, POST, or CAPSULE treatment period ${ }^{a, b}$

\begin{tabular}{|c|c|c|c|c|c|c|}
\hline & $\begin{array}{l}\mathrm{BL} \\
(n=30)\end{array}$ & $\begin{array}{l}\text { YS } \\
(n=25)\end{array}$ & $\begin{array}{l}\text { PRE } \\
(n=25)\end{array}$ & $\begin{array}{l}\text { POST } \\
(n=26)\end{array}$ & $\begin{array}{l}\text { CAP } \\
(n=26)\end{array}$ & $P$ Value \\
\hline$\overline{\mathrm{TC}, \mathrm{mg} / \mathrm{dL}}$ & $165 \pm 6$ & $167 \pm 6$ & $159 \pm 6$ & $162 \pm 6$ & $162 \pm 6$ & 0.45 \\
\hline $\mathrm{HDL}-\mathrm{C}, \mathrm{mg} / \mathrm{dL}$ & $54.2 \pm 2.0$ & $55.9 \pm 2.0$ & $55.7 \pm 2.0$ & $54.7 \pm 2.0$ & $54.2 \pm 2.0$ & 0.48 \\
\hline $\mathrm{LDL}-\mathrm{C}, \mathrm{mg} / \mathrm{dL}$ & $92.3 \pm 5.1$ & $93.5 \pm 5.1$ & $93.1 \pm 5.2$ & $91.9 \pm 5.2$ & $90.3 \pm 5.2$ & 0.71 \\
\hline Non-HDL-C, mg/dL & $110 \pm 6$ & $111 \pm 6$ & $111 \pm 6$ & $110 \pm 6$ & $108 \pm 6$ & 0.80 \\
\hline $\mathrm{TGs}, \mathrm{mg} / \mathrm{dL}$ & $90.4 \pm 6.3$ & $88.9 \pm 6.4$ & $86.8 \pm 6.4$ & $89.9 \pm 6.4$ & $88.8 \pm 6.4$ & 0.95 \\
\hline TC/HDL-C ratio & $3.15 \pm 0.14$ & $3.10 \pm 0.15$ & $3.13 \pm 0.15$ & $3.16 \pm 0.15$ & $3.12 \pm 0.15$ & 0.83 \\
\hline TG/HDL-C ratio & $1.77 \pm 0.14$ & $1.68 \pm 0.14$ & $1.69 \pm 0.14$ & $1.76 \pm 0.14$ & $1.75 \pm 0.14$ & 0.85 \\
\hline Insulin, $\mathrm{mU} / \mathrm{L}^{\mathrm{c}}$ & $5.06 \pm 0.88$ & $4.57 \pm 0.89$ & $5.50 \pm 0.90$ & $5.39 \pm 0.90$ & $4.69 \pm 0.90$ & 0.99 \\
\hline Glucose, $\mathrm{mg} / \mathrm{dL}^{\mathrm{c}}$ & $86.9 \pm 1.3$ & $87.9 \pm 1.3$ & $88.2 \pm 1.3$ & $87.1 \pm 1.3$ & $87.7 \pm 1.3$ & 0.74 \\
\hline HOMA-IR & $1.11 \pm 0.20$ & $1.00 \pm 0.20$ & $1.15 \pm 0.21$ & $1.19 \pm 0.21$ & $1.02 \pm 0.21$ & 0.63 \\
\hline $\mathrm{CRP}, \mathrm{mg} / \mathrm{L}^{\mathrm{c}}$ & $2.07 \pm 1.01$ & $1.77 \pm 1.05$ & $1.29 \pm 1.07$ & $2.17 \pm 1.07$ & $3.77 \pm 1.07$ & 0.99 \\
\hline
\end{tabular}

${ }^{a}$ Values are means \pm SEMs; BL, baseline; YS, yogurt smoothie with no BB-12 ${ }^{\circ}$; PRE, yogurt smoothie with BB- $12^{\circ}$ added pre-fermentation; POST, yogurt smoothie with $\mathrm{BB}-12^{\circ}$ post-fermentation; TC, total cholesterol; HDL-C, high-density lipoprotein cholesterol; LDL-C, low-density lipoprotein cholesterol; TGs, triglycerides; HOMA-IR, homeostatic model assessment-estimated insulin resistance; hs-CRP, high-sensitivity C-reactive protein

${ }^{\mathrm{b}}$ Statistical analyses were calculated using sex, treatment as between subject factor and randomization ID as the within-subject factor and age, BMI as covariates 'Statistical analyses were assessed by Kruskal-Wallis test

* $P$ values are for the main effect of treatment

influence the production of SCFAs. However, there is no evidence in the literature that the manufacturing process of yogurt smoothies influences the production of SCFAs. Therefore, further studies are warranted to elucidate effects of the manufacturing process of yogurt smoothies on the production of SCFAs.

The percentage change in acetate excretion was significantly higher after the YS period compared with the POST period (Fig. 2). This was an unexpected finding that acetate excretion increased after the YS period since $\mathrm{BB}-12^{\circ}$ was absent. Previous studies suggested that the production of SCFAs is influenced by prebiotics (indigestible carbohydrates) and probiotics (viable microorganisms that influence the gut microbial population) [31, 32]. In spite of the absence of probiotic in the YS, increased acetate excretion may come from ingredients in the yogurt smoothie, such as milk solids non-fat, strawberry puree, and pectin because SCFAs are produced from bacterial fermentation of polysaccharide, oligosaccharide, protein, peptide, and glycoprotein precursors [33].

In the present study, we found that the fecal excretion of SCFAs was negatively associated with blood lipids/lipoproteins (Table 4). Specifically, the levels of TC and LDL-C were negatively correlated with all of the fecal SCFAs. Our findings of the negative correlation between blood lipids/lipoproteins and SCFAs can be explained by previous in vitro and ex vivo studies that demonstrated that SCFAs suppress cholesterol synthesis by downregulating gene expression involved in intestinal cholesterol biosynthesis [34, 35]. Fushimi et al. showed that acetate influences serum cholesterol levels by decreasing hepatic 3-hydroxy-3-methylglutaryl-CoA synthase involved in cholesterol synthesis and increasing cholesterol $7 \alpha-$ hydroxylase involved in the conversion of cholesterol to bile acid in rats [36]. Consistent with this finding, a study by Fechner et al demonstrated that 4 weeks of lupin kernel fiber supplementation (25 g of lupin kernel

Table 6 Fecal SCFAs of participants at baseline and after 4 weeks of the YS, PRE, POST, or CAPSULE treatment period ${ }^{a, b}$

\begin{tabular}{lllllc}
\hline Variables & $\begin{array}{l}\mathrm{BL} \\
(n=30)\end{array}$ & $\begin{array}{l}\text { YS } \\
(n=25)\end{array}$ & $\begin{array}{l}\text { PRE } \\
(n=25)\end{array}$ & $\begin{array}{l}\text { POST } \\
(n=26)\end{array}$ & $\begin{array}{l}\text { CAP } \\
(n=26)\end{array}$ \\
\hline Total SCFAs, $\mu \mathrm{g} / \mathrm{g}$ & $2533 \pm 208$ & $2818 \pm 219$ & $2823 \pm 216$ & $2517 \pm 220$ & $2654 \pm 219$ \\
Acetate, $\mu \mathrm{g} / \mathrm{g}$ & $701 \pm 68$ & $914 \pm 71^{* *}$ & $900 \pm 70^{* *}$ & $773 \pm 71$ & $780 \pm 71$ \\
Propionate, $\mu \mathrm{g} / \mathrm{g}$ & $527 \pm 47$ & $633 \pm 50$ & $602 \pm 49$ & $529 \pm 50$ & $560 \pm 50$ \\
Butyrate, $\mu \mathrm{g} / \mathrm{g}$ & $798 \pm 86$ & $889 \pm 91$ & $921 \pm 90$ & $755 \pm 91$ & $865 \pm 91$ \\
\hline
\end{tabular}

${ }^{a}$ Values are means \pm SEMs; BL, baseline; YS, yogurt smoothie with no BB-12ं ; PRE, yogurt smoothie with BB- $12^{\circ}$ added pre-fermentation; POST, yogurt smoothie with $\mathrm{BB}-12^{\circ}$ post-fermentation; SCFAs, short chain fatty acids

${ }^{\mathrm{b}}$ Statistical analyses were calculated using sex, treatment as between subject factor and randomization ID as the within-subject factor and age, BMI, fiber intake as covariates

${ }^{*} P$ values are for the main effect of treatment

** Values are significantly difference from baseline $(P<0.01)$ 


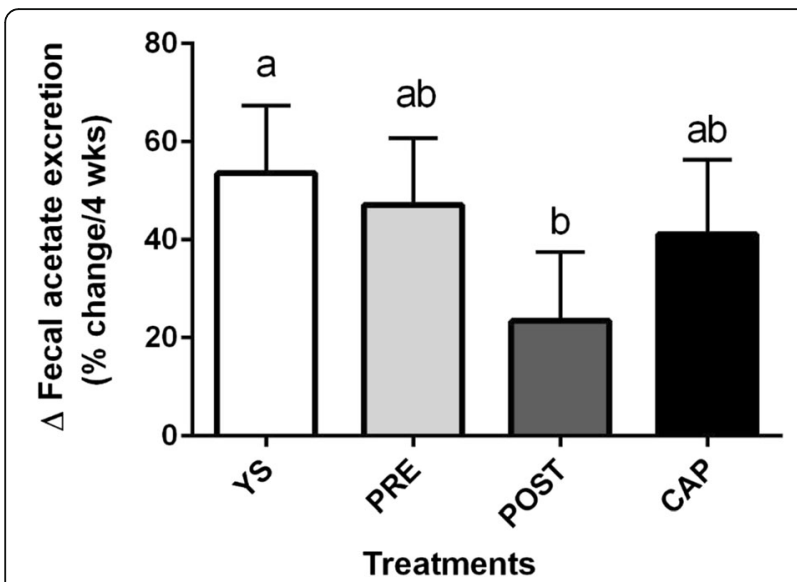

Fig. 2 Change (\%) in the excretion of fecal acetate after 4 weeks of the YS, PRE, POST, or CAPSULE treatment period. Bars are means of the percentage change. Different letters indicate significantly different values based on the mean of the square root transformed data. YS, yogurt smoothie with no BB-12; PRE, yogurt smoothie with BB- $12^{\circ}$ added pre-fermentation; POST, yogurt smoothie with BB-12 post-fermentation; SCFAs, short chain fatty acids

fiber/d) increased fecal SCFAs excretion and decreased the levels of TC (9\%) and LDL-C (12\%) in 60 hypercholesterolemic adults. The fecal excretion of SCFAs was negatively correlated with waist circumference. However, this finding was in contrast to other human trial data demonstrating that the fecal concentration of SCFAs was higher in overweight and obese individuals compared with lean individuals [37, 38]. It is noteworthy that our findings are based on exploratory analyses. Therefore, further studies are necessary in order to clarify the association between obesity and fecal SCFA concentrations. Another finding in our study is that age was inversely correlated with fecal concentration of SCFAs $\left(r_{\mathrm{p}}=-0.33, p<0.001\right)$. However, the effect of age on SCFAs is not fully understood. Yatsunenko et al [39] found that the human microbial population gradually decreases with increasing age, which may explain possible mechanisms for the inverse relationship between age and fecal SCFA concentrations.

There are strengths and limitations of our study. It was a randomized crossover study conducted with good compliance to daily BB- $12^{\circ}$ consumption for a relatively long study period (22 weeks). This study was novel because of the different delivery systems (yogurt vs. capsule) and manufacturing processes (pre vs. post fermentation) tested to assess how these factors may change the efficacy of the probiotic. However, it is unclear why fecal acetate concentrations were increased after the YS and PRE periods, but not after the POST period. In addition, our sample size was relatively small $(n=30)$ and predominantly Caucasian, which precludes extrapolating the findings to the population at large.
In conclusion, our study demonstrated that supplementation of BB-12 for 4 weeks did not improve lipids/ lipoproteins. We did not observe significant differences in total fecal SCFA concentrations between treatment periods. However, consumption of the yogurt smoothie increased the fecal acetate excretion compared to baseline. In addition, fecal SCFA concentrations were inversely related to TC and LDL-C, waist circumference and age. Thus, increasing fecal SCFAs may benefit cholesterol lowering, although older age and increased waist circumference may attenuate the response. Future studies are needed to better understand the association between fecal SCFA concentrations and TC, LDL-C, age, and waist circumference in young, healthy individuals.

\section{Abbreviations \\ BB-12: Bifidobacterium animalis subsp. lactis BB-12 ; BMI: Body mass index; CAP: BB- $12^{\circ}$ containing capsule; CRC: Clinical Research Center; CRP: C-reactive protein; CVD: Cardiovascular Disease; HDL-C: HDL cholesterol; HOMA- IR: Insulin resistance index; LDL-C: LDL cholesterol; METs: Metabolic equivalent of tasks; PRE: Yogurt smoothie with BB-12 ${ }^{\circ}$ added pre- fermentation; POST: Yogurt smoothie with BB-12 added post-fermentation; SCFA: Short Chain Fatty Acids; TC: Total cholesterol; TGs: Triglycerides; YS: Yogurt smoothie with no BB- $12^{\circ}$}

\section{Acknowledgements}

We thank our study participants for their dedication to this study. We also thank staff nurses at the Penn State Clinical Research Center for their collaboration.

\section{Funding}

This research was supported by a grant from The Dairy Research Institute.

\section{Availability of data and materials}

The datasets generated during the current study are available from the corresponding author on reasonable request.

\section{Authors' contributions}

RFR, PMKE, and CJR designed research; YL, ZB, JAF, EJF and HM conducted research; $Y L$ performed statistical analysis; $Y L$ wrote the paper. PMKE had primary responsibility for final content. All authors read and approved the final manuscript.

\section{Ethics approval and consent to participate}

The study protocol was approved by the Institutional Review Board of The Pennsylvania State University. Written informed consent was obtained from all enrolled participants at the screening visit.

\section{Consent for publication}

Not applicable.

\section{Competing interests}

The authors declare that they have no competing interests.

\section{Publisher's Note}

Springer Nature remains neutral with regard to jurisdictional claims in published maps and institutional affiliations.

\section{Author details}

${ }^{1}$ Department of Nutritional Sciences, Pennsylvania State University, 110 Chandlee Laboratory, University Park, PA 16802, USA. ²Department of Food Science, Pennsylvania State University, 206 Rodney A. Erickson Food Science Building, University Park, PA 16802, USA. 
Received: 4 January 2017 Accepted: 20 June 2017

Published online: 29 June 2017

\section{References}

1. Mozaffarian D, Benjamin EJ, Go AS, Arnett DK, Blaha MJ, Cushman M, Das SR, de Ferranti S, Despres JP, Fullerton HJ, et al. Executive Summary: Heart Disease and Stroke Statistics-2016 Update: A Report From the American Heart Association. Circulation. 2016;133:447-54.

2. Ference BA, Ginsberg HN, Graham I, Ray KK, Packard CJ, Bruckert E, Hegele RA, Krauss RM, Raal FJ, Schunkert H, et al. Low-density lipoproteins cause atherosclerotic cardiovascular disease. 1. Evidence from genetic, epidemiologic, and clinical studies. A consensus statement from the European Atherosclerosis Society Consensus Panel. Eur Heart J. 2017.

3. Lebeer S, Vanderleyden J, De Keersmaecker SC. Host interactions of probiotic bacterial surface molecules: comparison with commensals and pathogens. Nat Rev Microbiol. 2010;8:171-84.

4. Osterlund P, Ruotsalainen T, Korpela R, Saxelin M, Ollus A, Valta P, Kouri M, Elomaa I, Joensuu H. Lactobacillus supplementation for diarrhoea related to chemotherapy of colorectal cancer: a randomised study. Br J Cancer. 2007; 97:1028-34.

5. Salminen E, Elomaa I, Minkkinen J, Vapaatalo H, Salminen S. Preservation of intestinal integrity during radiotherapy using live Lactobacillus acidophilus cultures. Clin Radiol. 1988;39:435-7.

6. Dinleyici EC, Dalgic N, Guven S, Metin O, Yasa O, Kurugol Z, Turel O, Tanir G, Yazar AS, Arica V, et al. Lactobacillus reuteri DSM 17938 shortens acute infectious diarrhea in a pediatric outpatient setting. J Pediatr (Rio J). 2015;91:392-6.

7. Lee do K, Park JE, Kim MJ, Seo JG, Lee JH, Ha NJ. Probiotic bacteria, B. longum and L. acidophilus inhibit infection by rotavirus in vitro and decrease the duration of diarrhea in pediatric patients. Clin Res Hepatol Gastroenterol. 2015;39:237-44.

8. Strisciuglio C, Miele E, Giugliano FP, Vitale S, Andreozzi M, Vitale A, Catania MR, Staiano A, Troncone R, Gianfrani C. Bifidobacteria Enhance Antigen Sampling and Processing by Dendritic Cells in Pediatric Inflammatory Bowel Disease. Inflamm Bowel Dis. 2015;21:1491-8.

9. Jespersen L, Tarnow I, Eskesen D, Morberg CM, Michelsen B, Bugel S, Dragsted LO, Rijkers GT, Calder PC. Effect of Lactobacillus paracasei subsp. paracasei, L. casei 431 on immune response to influenza vaccination and upper respiratory tract infections in healthy adult volunteers: a randomized, double-blind, placebo-controlled, parallel-group study. Am J Clin Nutr. 2015;101:1188-96.

10. Meng H, Ba Z, Lee Y, Peng J, Lin J, Fleming JA, Furumoto EJ, Roberts RF, Kris-Etherton PM, Rogers CJ. Consumption of Bifidobacterium animalis subsp. lactis BB-12 in yogurt reduced expression of TLR-2 on peripheral blood-derived monocytes and pro-inflammatory cytokine secretion in young adults. Eur J Nutr. 2017;56(2):649-61.

11. Begley M, Hill C, Gahan CG. Bile salt hydrolase activity in probiotics. Appl Environ Microbiol. 2006;72:1729-38.

12. Pereira DI, McCartney AL, Gibson GR. An in vitro study of the probiotic potential of a bile-salt-hydrolyzing Lactobacillus fermentum strain, and determination of its cholesterol-lowering properties. Appl Environ Microbiol. 2003;69:4743-52.

13. Pereira DI, Gibson GR. Cholesterol assimilation by lactic acid bacteria and bifidobacteria isolated from the human gut. Appl Environ Microbiol. 2002; 68:4689-93.

14. DiRienzo DB. Effect of probiotics on biomarkers of cardiovascular disease: implications for heart-healthy diets. Nutr Rev. 2014;72:18-29.

15. Bertolami MC, Faludi AA, Batlouni M. Evaluation of the effects of a new fermented milk product (Gaio) on primary hypercholesterolemia. Eur J Clin Nutr. 1999:53:97-101.

16. Ejtahed HS, Mohtadi-Nia J, Homayouni-Rad A, Niafar M, Asghari-Jafarabadi M, Mofid V, Akbarian-Moghari A. Effect of probiotic yogurt containing Lactobacillus acidophilus and Bifidobacterium lactis on lipid profile in individuals with type 2 diabetes mellitus. J Dairy Sci. 2011;94:3288-94.

17. Agerbaek M, Gerdes LU, Richelsen B. Hypocholesterolaemic effect of a new fermented milk product in healthy middle-aged men. Eur J Clin Nutr. 1995; 49:346-52.

18. Guardamagna O, Amaretti A, Puddu PE, Raimondi S, Abello F, Cagliero P, Rossi M. Bifidobacteria supplementation: effects on plasma lipid profiles in dyslipidemic children. Nutrition. 2014;30:831-6.

19. Ivey $\mathrm{KL}$, Hodgson JM, Kerr DA, Thompson PL, Stojceski B, Prince RL. The effect of yoghurt and its probiotics on blood pressure and serum lipid profile; a randomised controlled trial. Nutr Metab Cardiovasc Dis. 2015;25:46-51.

20. Greany KA, Bonorden MJ, Hamilton-Reeves JM, McMullen MH, Wangen KE, Phipps WR, Feirtag J, Thomas W, Kurzer MS. Probiotic capsules do not lower plasma lipids in young women and men. Eur J Clin Nutr. 2008;62:232-7.

21. de Roos NM, Schouten G, Katan MB. Yoghurt enriched with Lactobacillus acidophilus does not lower blood lipids in healthy men and women with normal to borderline high serum cholesterol levels. Eur J Clin Nutr. 1999:53:277-80.

22. Miller TL, Wolin MJ. Fermentations by saccharolytic intestinal bacteria. Am J Clin Nutr. 1979;32:164-72.

23. Cummings $\mathrm{JH}$. Fermentation in the human large intestine: evidence and implications for health. Lancet. 1983;1:1206-9.

24. Topping DL, Clifton PM. Short-chain fatty acids and human colonic function: roles of resistant starch and nonstarch polysaccharides. Physiol Rev. 2001;81:1031-64.

25. Wang J, Zhang H, Chen X, Chen Y, Menghebilige, Bao Q. Selection of potential probiotic lactobacilli for cholesterol-lowering properties and their effect on cholesterol metabolism in rats fed a high-lipid diet. J Dairy Sci. 2012;95:1645-54.

26. Merenstein D, Gonzalez J, Young AG, Roberts RF, Sanders ME, Petterson S. Study to investigate the potential of probiotics in children attending school. Eur J Clin Nutr. 2011;65:447-53.

27. Garcia-Villalba R, Gimenez-Bastida JA, Garcia-Conesa MT, Tomas-Barberan FA Carlos Espin J, Larrosa M. Alternative method for gas chromatography-mass spectrometry analysis of short-chain fatty acids in faecal samples. J Sep Sci. 2012;35:1906-13.

28. Bouchard C, Tremblay A, Leblanc C, Lortie G, Savard R, Theriault G. A method to assess energy expenditure in children and adults. Am J Clin Nutr. 1983;37:461-7.

29. Jones ML, Martoni CJ, Prakash S. Cholesterol lowering and inhibition of sterol absorption by Lactobacillus reuteri NCIMB 30242: a randomized controlled trial. Eur J Clin Nutr. 2012;66:1234-41.

30. Agerholm-Larsen L, Raben A, Haulrik N, Hansen AS, Manders M, Astrup A. Effect of 8 week intake of probiotic milk products on risk factors for cardiovascular diseases. Eur J Clin Nutr. 2000;54:288-97.

31. Brighenti F, Testolin G, Canzi E, Ferrari A, Wolever TMS, Ciappellano S, Porrini $M$, Simonetti P. Influence of long-term feeding of different purified dietary fibers on the volatile fatty acid (VFA) profile, $\mathrm{pH}$ and fiber-degrading activity of the cecal contents in rats. Nutr Res. 1989;9:761-72.

32. Kim HK, Rutten NB, Besseling-van der Vaart I, Niers LE, Choi YH, Rijkers GT, van Hemert S. Probiotic supplementation influences faecal short chain fatty acids in infants at high risk for eczema. Benef Microbes. 2015;6:783-90.

33. Cummings $\mathrm{JH}$, Hill MJ, Jivraj T, Houston $H$, Branch WJ, Jenkins DJ. The effect of meat protein and dietary fiber on colonic function and metabolism. I. Changes in bowel habit, bile acid excretion, and calcium absorption. Am J Clin Nutr. 1979;32:2086-93.

34. Hara H, Haga S, Aoyama Y, Kiriyama S. Short-chain fatty acids suppress cholesterol synthesis in rat liver and intestine. J Nutr. 1999;129:942-8.

35. Alvaro A, Sola R, Rosales R, Ribalta J, Anguera A, Masana L, Vallve JC. Gene expression analysis of a human enterocyte cell line reveals downregulation of cholesterol biosynthesis in response to short-chain fatty acids. IUBMB Life. 2008;60:757-64.

36. Fushimi T, Suruga K, Oshima Y, Fukiharu M, Tsukamoto Y, Goda T. Dietary acetic acid reduces serum cholesterol and triacylglycerols in rats fed a cholesterol-rich diet. Br J Nutr. 2006;95:916-24.

37. Rahat-Rozenbloom S, Fernandes J, Gloor GB, Wolever TM. Evidence for greater production of colonic short-chain fatty acids in overweight than lean humans. Int J Obes (Lond). 2014:38:1525-31.

38. Schwiertz A, Taras D, Schafer K, Beijer S, Bos NA, Donus C, Hardt PD. Microbiota and SCFA in lean and overweight healthy subjects. Obesity (Silver Spring). 2010;18:190-5.

39. Yatsunenko T, Rey FE, Manary MJ, Trehan I, Dominguez-Bello MG, Contreras M, Magris M, Hidalgo G, Baldassano RN, Anokhin AP, et al. Human gut microbiome viewed across age and geography. Nature. 2012;486:222-7. 